\title{
Fatores associados à experiência de cárie em escolares de um município com baixa prevalência de cárie dentária
}

\author{
Factors associated with The Incidence of Dental Caries \\ Among Schoolchildren Living In A Municipality \\ With Low Prevalence of Dental Caries
}

\author{
Silvia Cypriano ${ }^{1}$ \\ Fernando N eves Hugo ${ }^{2}$ \\ M aria Cristina Sciamarelli ${ }^{3}$ \\ Luísa H elena do N ascimento Tôrres ${ }^{4}$ \\ M aria da Luz Rosário de Sousa ${ }^{5}$ \\ Ronaldo Seichi Wada ${ }^{5}$
}

\footnotetext{
1 Programa de PósGraduação em Odontologia área de Cariologia, nível Doutorado, Faculdadede Odontologia dePiracicaba, UniversidadeEstadual de Campinas. Caixa postal $\mathrm{n}$. 52. 13414-903 Piracicaba SP.

silviacypriano@hotmail.com ${ }^{2}$ Departamento de

Odontologia Preventivae

Social, Faculdadede

Odontologia, U niversidade

Federal do Rio Grandedo Sul

${ }^{3}$ Saúde Bucal, Secretaria Municipal deSaúde, Prefeitura M unicipal de Jundiá

4 Programa de Pós-

Graduação em Odontologia, área deSaúdeColetiva, nível M estrado, Faculdadede Odontologia de Piracicaba, UniversidadeEstadual de Campinas

${ }^{5}$ Departamento de

Odontologia Social, FaculdadedeO dontologia de Piracicaba, Universidade Estadual deCampinas
}

Abstract The literature has shown that poorer levels of oral health are more frequently related to lower socio-economic status, consequently this cross-sectional and exploratory study conducted in 2003 investigated the association between caries and socio-economic factors, access to care, selfperception and habits among 266 12-year-old schoolchildren living in a community with low prevalence of dental caries. World Health Organization dental caries diagnosis methodology was used, in addition to the application of socio- economic and behavioral questionnaires. To identify the factors associated with dental caries, multivariate logistic regression was used and the dependent variable was synthesized into DM FT $=0$ and $\mathrm{DMFT}>0$. Bearing in mind the limitations of a cross-sectional study, disliking the appearance of teeth, seeking dental care because of pain, studying at a state school and the head of the family beinga manual worker wereindependently associated with dental caries. Even in a municipality with low prevalence of caries, the socioeconomic status, dental care and self-perception were important factors in the incidence of dental caries among schoolchildren, and it is recommended that many factors in the bio-psychosocial context of multi-factorial dental caries should be investigated.

Key words Dental caries, DM FT Index, Oral health, Socio-economic factors
Resumo A literatura tem mostrado que piores níveis de saúde bucal estão mais frequentemente relacionados a baixos níveis socioeconômicos, assim este estudo transversal de caráter exploratório investigou a associação entre experiência de cárie e fatores socioeconômicos, acesso a serviços, autopercepção e hábitos em 266 escolares de 12 anos de idade de uma localidade com baixa pre valência de cárie dentária em 2003. Utilizou-se critérios de diagnóstico para cárie dentária da Organização M undial da Saúdeeaplicou-sequestionário socioecômico e comportamental. Para a identificação dos fatores associados à experiência de cárie utilizou-sea análise de regressão logística multivariada. A variável dependente foi dicotomizada em $C P O D=0$ e CPOD $>0$. Considerando as limitações de um estudo transversal, não gostar da aparência dos dentes, procurar atendimento odontológico por dor, estudar em escola pública e o responsável da família ser trabalhador manual foram independentemente associados à experiência de cárie. M esmo em um município com baixa prevalência de cárie os fatores socioeconômicos, 0 acesso a serviço odontológico e a autopercepção da saúde bucal foram importantes para a ocorrência da cárie, recomendando-se que diversos fatores sejam investigados dentro do contexto biopsicossocial da sua multifatoriedade.

Palavras-chave Cárie dentária, Índice CPO, Saúde bucal, Fatores socioeconômicos 
Introdução

A evidente desigualdade na nossa sociedade das condições de saúde e de acesso aos serviços pode ser enten dida como um reflexo de fatores relacionados às condições socioeconômicas e aos padrões culturais dos indivíduos e não podem ser inteiramente explicados por cuidados médicos, fatores genéticos ou pelo comportamento dos indivíduos ${ }^{1}$. As reais dimensões destas desigualdades são ainda pouco conhecidas na América Latina e Caribe ${ }^{2}$.

Assim, vários estudos científicos verificaram uma associação entre a experiência de cárie dentária e as condições relacionadas ao nível socioe conômico, tanto nos estudos baseados no indivíduo ${ }^{3-9}$ como nos ecológicos ${ }^{10-17}$, ou seja, as condições de saúde bucal são piores nos grupos sociais menosfavorecidoseconomicamente. Entre tanto, este padrão não é universal, pois alguns estudos de países em desenvolvimento na África demonstraram que as crianças que pertenciam a classes sociais mais altas têm mais cáries que as aquelas pertencentes a classes sociais mais baixas, sendo invertida esta relação em países de senvolvidos ${ }^{18,19}$.

Considerando o atual contexto epidemiológico da prevalência da cárie dentária no Brasil, em que se observa uma importante redução na média de dentes atacados pela cárie em populações jovens ${ }^{20-22}$ e um aumento na proporção de crianças sem experiência de cárie ${ }^{23-26}$, torna-se fundamental a investigação dos fatores socioe conômicos e comportamentais associados a este fenômeno de uma forma diferenciada de acordo com as regras em quea doença ocorre. Como no caso do presente estudo, que trata de um município de baixa prevalência, pois a partir deste conhecimento seria possível uma maior adequação de programas e políticas de saúde para minimizar as iniquidades em saúde bucal.

Assim, o objetivo deste estudo foi analisar os fatores socioeconômicos, de acesso aos serviços, autopercepção e hábitos associados com a expe riência da cárie dentária em escolares de 12 anos de idadeem uma localidadecom baixa prevalência deste problema.

\section{M etodologia}

No ano de 2003, realizou-se um estudo do tipo transversal para avaliar a prevalência e a distribuição da cárie dentária em escolares de 12 anos de idade (CPO-D = 2,3 aos 12 anos, considerada pela OM S como de baixa prevalência), matriculados na rede pública e privada de ensino da cidade de Jundiaí (SP). Trata-se de um município de grande porte, com uma população estimada em 341 mil habitantes, alto Í ndice de Desenvolvimento Humano (IDH-M de0,857 em 2000), sendo superior à média do Estado de São Paulo $(0,814)$, alta taxa de urbanização $(93,8 \%)$, além de apresentar bons indicadores de saúde como, por exemplo, a taxa de mortalidade infantil em 2002 ter sido de 9,92/mil crianças nascidas vivase a longevidade de 68 anos $^{27}$.

0 município também apresenta alta cobertura de saneamento básico, e 97,3\% da população recebe água tratada e fluoretada desde 1980. A Secretaria de Saúde do M unicípio realiza sistematicamente 0 heterocontrole da fluoretação, através do Programa Pró-Água, sendo que 97,6\% das amostras analisadas apresentaram-se com valores oscilando entre 0,6 a $0,8 \mathrm{ppm}$ defluoreto no ano de 2003. A maioria dos escolares que foram investigados residia no município desde 0 nascimento $(77,8 \%)$.

Para este estudo utilizou-se dados secundários, sendo o mesmo aprovado pelo Comitê de Ética da Faculdade de Odontologia de Piracicaba/ UNICAMP.

\section{Amostra}

Para o cálculo do tamanho da amostra utilizou-se o valor da média do índice CPOD $(4,1)$ e desvio-padrão $(5,3)$ obtidos em um estudo realizado no ano de $1998^{28}$ adotando-se para tal nível de confiança de $95 \%$, precisão de $10 \%$ e efeito do desenho (deff) de $2^{29}$. Somaram-se $20 \%$ à amostra, a fim de compensar eventuais perdas e recusas, totalizando assim 333 crianças.

Foi utilizado o processo de amostragem em duplo estágio: as escolas públicas e as privadas como unidades do primeiro estágio e os alunos como unidades do segundo estágio. Assim, identificou-se todos os estabelecimentos de ensino do município frequentados por crianças de 12 anos (12 anos completos até outubro de 2003) por meio de listagens fornecidas pela Secretaria Municipal de Ensino, sorteando-se 20 escolas de ensino fundamental para a seguir proceder ao sorteio dos el ementos amostrais, utilizando a técnica da amostragem sistemática.

Enviou-se aos pais ou responsáveis das crianças sorteadas o termo de consentimento livre e esclarecido, para a autorização dos exames epidemiológicos. Em anexo encaminhou-se um questionário para que se obtivessem informa- 
ções das variáveis de interesse, compondo quatro grupos distintos: 1) I dentificação da criança (local de nascimento, gênero, idade); 2) Variáveis de nível socioeconômico (profissão e anos de estudo do responsável pela família, número de cômodos, tipo de moradia, número de apareIhos de tel evisão, possee número de automóveis, sea família possui plano de saúdeou plano odontológico e número de pessoas que residem na casa); 3) Acesso a serviços odontológicos (tipo de serviço utilizado, acesso e motivo da consulta); 4) hábitos (frequência de escovação diária, supervisão da escovação, tipo de água consumida com maior frequência, faltar na escola por motivo de dor e acesso a orientações de saúde bucal). Estequestionário teve como referencial o roteiro de entrevistas do Projeto SB Brasi ${ }^{30}$, fazendo-se as modificações necessárias para seadequar aos objetivos deste estudo, assim como a faixa etária de interesse. Foi aplicado um piloto em uma das escolas que não participou da amos$\operatorname{tra}(n=38)$.

Uma das variáveis utilizadas para a determinação do nível socioeconômico das crianças foi a profissão do responsável pela família. Esta variável foi classificada de acordo com o Registro Geral de Categorias Profissionais do Reino Uni$\mathrm{do}^{31}$, sendo posteriormente categorizado como trabalhadores manuais e não manuais.

Durante o exame clínico incluíram-se duas questões relacionadas à autopercepção:

Você gosta da aparência dos seus dentes?

Você sentiu alguma dor quando comeu ou mastigou nos últimos 06 meses?

Treinamento da equipe de examinadores e concordância interexaminadores

0 processo de calibração foi realizado em 40 horas, próximo à fase de coleta dos dados, com discussões teóricas e atividades práticas simulando as diferentes condições esituações quea equipe de examinadores encontraria durante a realização da coleta de dados. A equipe foi constituída por 05 examinadores, obtendo-se uma reprodutibilidade diagnóstica para a cárie dentária acima de 0,83 do índice kappa.

Exame das condições de saúde bucal e índices utilizados

Os exames clínicosforam realizados com iluminação natural, utilizando-sesondas CPI (ballpoint) e espelhos bucais planos. A cárie dentária foi avaliada tendo como base os critérios de di- agnóstico recomendados pela Organização M undial da Saúde (OM S) ${ }^{32}$. Outras condições desaúde bucal foram pesquisadas, entretanto não serão abordadas no presente estudo.

Coleta dos dados e desfecho estudado

A coleta dos dados ocorreu nos meses de novembro e dezembro de 2003, e aproximadamente $9 \%$ da amostra foi reexaminada durantea fase de coleta dos dados. Os valores de concordância obtidos foram acima de $98 \%$ e o índice kappa variou de 0,98 a 0,94, para a condição dental, mostrando que os níveis de reprodutibilidade diagnóstica intraexaminador foram mantidos durante esta etapa do estudo. 0 desfecho estudado foi a experiência de cárie, analisada através da variável dependente CPOD, ou seja, CPOD $=0$ $(Y=0)$ ou $C P O D>0(Y=1)$.

\section{Processamento dos dados}

e análise estatística

Para a elaboração do banco de dados utilizou-se software Epi-Info. Posteriormente, realizou-se a estatística descritiva da prevalência da cárie dentária deacordo com as variáveis deinteresse, adotando-se o nível designificância de $5 \%$. Comparou-se o tipo de escola (pública ou privada), por meio do teste do Qui-quadrado $\left(\chi^{2}\right)$ e $M$ ann-W hitney Test. A adoção de testes não paramétricoséjustificada, poiso índice CPOD não apresentou distribuição normal na amostra estudada $(p<0,001)$.

A seguir foram realizados cálculos da distribuição das frequências de todas as variáveis independentes, em relação ao desfecho estudado. As categorias das variáveis queapresentaram distribuição semelhante foram agrupadas para a realização dos testes de associação do Qui-quadrado e as análises univariadas, estimando-se assim as razões de probabilidade (Odds Ratio OR) eos respectivos intervalos de confiança (IC) de $95 \%$. Todas as variáveis independentes mais fortemente relacionadas $(p<0,25)$, bem como aquelas que são epidemiologicamente relevantes, de acordo com dados da literatura, em relação ao desfecho estudado, foram incluídas na análise logística multivariada, utilizando-se para tal o programaSPSS, versão 9.0. As variáveis que não contribuíram para o modelo foram eliminadas e um novo foi calculado. A significância estatística foi avaliada através do teste da razão de verossimilhança (Likelihood Ratio Test). 
Resultados

A amostra contou com 266 escolares, sendo 123 (46,24\%) do gênero masculino e $143(53,76 \%)$ do feminino. Entre os escolares, $73,7 \%$ estudavam em escola pública e $26,3, \%$ em escola privada, sendo $47 \%$ da amostra livre de cárie.

A taxa de resposta obtida neste estudo foi de $80,2 \%$, sendo queas perdas se deveram principalmente a não autorização dos pais, questionários incompletos ou sem respostas e a criança não estar presente na escola no momento do exame.

A estatística descritiva para a cárie dentária, segundo gênero, tipo de escola e a sua localização, pode ser observadas na Tabela 1, sendo verificada uma melhor situação de saúde bucal em estudantes deescolas privadas eresidentes naárea urbana do município, não apresentando diferenças quanto ao gênero.

Com a finalidade de identificar os fatores relacionados à presença ou não de cárie dentária, foi realizado o teste de Qui-quadrado entre a variável dependente dicotômica (CPOD) eas variáveis independentes (Tabela 2), ressaltando-se que as variáveis tipo e localização da escola, tipo de moradia, número de cômodos e de aparelhos de televisão, profissão e anos de estudo do responsável pela família, bem como itens de acesso a serviços de saúde bucal e de autopercepção/ hábitos apresentaram relação com CPOD >0.

Realizou-se, então, a análise univariada das variáveis que apresentaram significância estátís- tica pelo teste do Qui-Quadrado $(p<0,05)$, podendo ser observados o 0 dds Ratio e o Intervalo de Confiança de cada categoria na Tabela 3, após os agrupamentos realizados, verificando-se que as variáveis do nível socioeconômico, de acesso a serviços de saúde bucal e de autopercepção/hábitos mantiveram-se significativas.

Para a elaboração do modelo de regressão logística múltiplo, inseriram-se as variáveis com maior significância estatística $(p<0,25)$. 0 modelo final pode ser observado na Tabela 4. Os resultados encontrados após a análise mostraram que a criança não gostar da aparência dos seus dentes, ter procurado atendimento por motivo de dor, o responsável pela família ser trabaIhador manual e estudar em escola pública foram fatores associados significativos para o desfecho experiência de cárie, após o ajuste das demais variáveis.

\section{Discussão}

Este estudo transversal de caráter exploratório contribuiu para uma discussão mais abrangente sobre a relação entre a saúde bucal e os fatores associados à doença cárie, pois encontrou importantes associações entreexperiência decáriee fatores socioeconômicos eacesso a serviços, além de investigar hábitos, tendo como base o roteiro deentrevistas utilizado na Pesquisa SB Brasil, com adaptações específicas para este estudo.

Tabela 1. Experiência e distribuição da cárie dentária aos 12 anos de idade, segundo o gênero, o tipo e a localização da escola. Jundiaí, 2003.

\begin{tabular}{|c|c|c|c|c|c|}
\hline & \multicolumn{2}{|c|}{ Com experiência de cárie } & \multicolumn{3}{|c|}{ Distribuição da cárie } \\
\hline & $\mathrm{n}(\%)$ & CPOD (dp) & $\begin{array}{c}C P O D=0 \\
n(\%)\end{array}$ & $\begin{array}{c}\text { CPOD 1-4 } \\
\text { n (\%) }\end{array}$ & $\begin{array}{c}\text { CPOD 5-11 } \\
n(\%)\end{array}$ \\
\hline \multicolumn{6}{|l|}{ Gênero } \\
\hline M asculino & $65(52,8)^{a}$ & $1,5^{\mathrm{a}}(1,8)$ & $58(47,2)^{a}$ & $54(43,9)^{a}$ & $11(8,9)^{a}$ \\
\hline Feminino & $76(53,1)^{a}$ & $1,9^{\mathrm{a}}(2,4)$ & $67(46,9)^{a}$ & $54(37,8)^{a}$ & $22(15,2)^{\mathrm{a}}$ \\
\hline Valor de $p$ & 0,961 & 0,397 & 0,961 & 0,309 & 0,112 \\
\hline \multicolumn{6}{|l|}{ Tipo de escola } \\
\hline Pública (n=196) & $115(58,7)^{\mathrm{a}}$ & $1,9^{\mathrm{a}}(2,3)$ & $81(41,3)^{a}$ & $85(43,4)^{a}$ & $30(15,3)^{a}$ \\
\hline Privada $(n=70)$ & $26(37,1)^{b}$ & $1,2^{b}(1,7)$ & $44(62,9)^{b}$ & $23(32,9)^{a}$ & $3(4,2)^{b}$ \\
\hline Valor de $p^{*}$ & 0,002 & 0,008 & 0,002 & 0,124 & 0,016 \\
\hline \multicolumn{6}{|l|}{ Localização da escola } \\
\hline Área urbana $(n=223)$ & $112(50,2)^{a}$ & $1,6^{\mathrm{a}}(2,0)$ & $111(49,8)^{a}$ & $87(39,0)^{a}$ & $25(11,2)^{a}$ \\
\hline Área rural $(n=43)$ & $29(67,4)^{b}$ & $2,4^{b}(2,6)$ & $14(32,6)^{b}$ & $21(48,8)^{a}$ & $8(18,6)^{a}$ \\
\hline Valor de p* $^{*}$ & 0,038 & 0,042 & 0,038 & 0,230 & 0,178 \\
\hline Total $(n=226)$ & $141(53,0)$ & $1,7(2,1)$ & $125(47,0)$ & $108(40,6)$ & $33(12,4)$ \\
\hline
\end{tabular}

Números seguidos de letras sobrescritas na vertical diferem entre si com significância de 5\%; * Teste Q ui-quadrado foi utilizado para verificar se havia diferenças entre as proporçõese o $M$ ann-W hitney test para as médias. 
Tabela 2. Teste de Q ui-Quadrado $\left(\chi^{2}\right)$ e valores de $p$ segundo variáveis de identificação da criança, nível sócio-econômico, acesso a serviços de saúde, autopercepção e hábitos comportamentais em escolares de 12 anos de idade. Jundiaí (SP), 2003.

\begin{tabular}{|c|c|c|c|c|c|c|}
\hline \multirow[b]{2}{*}{ Variáveis } & \multicolumn{2}{|c|}{$C P O D=0$} & \multicolumn{2}{|c|}{$C P O D>0$} & \multirow[b]{2}{*}{$\chi^{2}$} & \multirow[b]{2}{*}{ Valores de $p^{* *}$} \\
\hline & $\mathrm{N}^{*}$ & $\%$ & $\mathrm{~N}^{*}$ & $\%$ & & \\
\hline \multicolumn{7}{|l|}{ 1. Identificação } \\
\hline \multicolumn{7}{|l|}{ Gênero } \\
\hline Masculino & 58 & 47,2 & 65 & 52,8 & 0,02 & 0,961 \\
\hline Feminino & 67 & 46,9 & 76 & 53,1 & & \\
\hline \multicolumn{7}{|l|}{ Etnia } \\
\hline Branco & 62 & 47,3 & 69 & 52,7 & 5,48 & 0,140 \\
\hline Negro & 12 & 52,2 & 11 & 47,8 & & \\
\hline Pardo & 45 & 42,9 & 60 & 57,1 & & \\
\hline Amarelo & 4 & 100 & 0 & 0,0 & & \\
\hline \multicolumn{7}{|c|}{ Reside no município desde o nascimento } \\
\hline Sim & 97 & 46,9 & 110 & 53,1 & 0,001 & 0,935 \\
\hline Não & 28 & 47,5 & 31 & 52,5 & & \\
\hline \multicolumn{7}{|l|}{ 2. Nível socioeconômico } \\
\hline \multicolumn{7}{|l|}{ Tipo de escola } \\
\hline Privada & 44 & 62,9 & 26 & 37,1 & 9,60 & 0,002 \\
\hline Pública & 81 & 41,3 & 115 & 58,7 & & \\
\hline \multicolumn{7}{|l|}{ Localização da escola } \\
\hline Urbana & 111 & 49,8 & 112 & 50,2 & 4,29 & 0,038 \\
\hline Rural & 14 & 32,9 & 29 & 67,4 & & \\
\hline \multicolumn{7}{|l|}{ Tipo de M oradia } \\
\hline Própria & 83 & 51,6 & 78 & 48,4 & 6,58 & 0,037 \\
\hline Alugada & 20 & 51,3 & 19 & 48,7 & & \\
\hline Cedida & 22 & 33,3 & 44 & 66,7 & & \\
\hline \multicolumn{7}{|l|}{$\mathrm{N}^{\circ}$ de pessoas residentes } \\
\hline$<4$ & 69 & 53,9 & 59 & 46,1 & 5,48 & 0,065 \\
\hline 5 ou 6 & 44 & 42,7 & 59 & 57,3 & & \\
\hline$>7$ & 12 & 34,3 & 23 & 65,7 & & \\
\hline \multicolumn{7}{|l|}{$\mathrm{N}^{\circ}$ de cômodos no domicilio } \\
\hline$>6$ & 46 & 58,2 & 33 & 41,8 & 5,69 & 0,017 \\
\hline$<6$ & 79 & 42,2 & 108 & 57,8 & & \\
\hline \multicolumn{7}{|l|}{$\mathrm{N}^{\circ}$ de aparelhos de televisão } \\
\hline$>2$ & 79 & 57,7 & 58 & 42,3 & 12,92 & 0,000 \\
\hline$<2$ & 46 & 35,7 & 83 & 64,3 & & \\
\hline \multicolumn{7}{|l|}{ Posse de automóvel } \\
\hline Sim & 68 & 50,7 & 66 & 49,3 & 1,53 & 0,216 \\
\hline Não & 57 & 43,2 & 75 & 56,8 & & \\
\hline \multicolumn{7}{|l|}{ Plano de saúde familiar } \\
\hline Sim & 38 & 52,1 & 35 & 47,9 & 1,04 & 0,309 \\
\hline Não & 87 & 45,1 & 106 & 54,9 & & \\
\hline Profissão do responsável pel & & & & & & \\
\hline Não manual & 54 & 60,7 & 35 & 39,3 & 10,05 & 0,002 \\
\hline Manual & 71 & 40,1 & 106 & 59,9 & & \\
\hline Anos de estudo do responsá & & & & & & \\
\hline$>8$ & 59 & 56,7 & 45 & 43,3 & 6,50 & 0,011 \\
\hline$<8$ & 66 & 40,7 & 96 & 59,3 & & \\
\hline 3. Acesso a serviços de Saúde B & & & & & & \\
\hline Acesso ao dentista & & & & & & \\
\hline Sim & 107 & 45,7 & 127 & 54,3 & 1,25 & 0,263 \\
\hline Não & 18 & 56,3 & 14 & 43,8 & & \\
\hline Tipo de serviço & & & & & & \\
\hline Privado & 62 & 57,9 & 45 & 42,1 & 12,92 & 0,002 \\
\hline Convênio & 28 & 50,9 & 27 & 49,1 & & \\
\hline Público & 35 & 33,7 & 69 & 66,3 & & \\
\hline
\end{tabular}




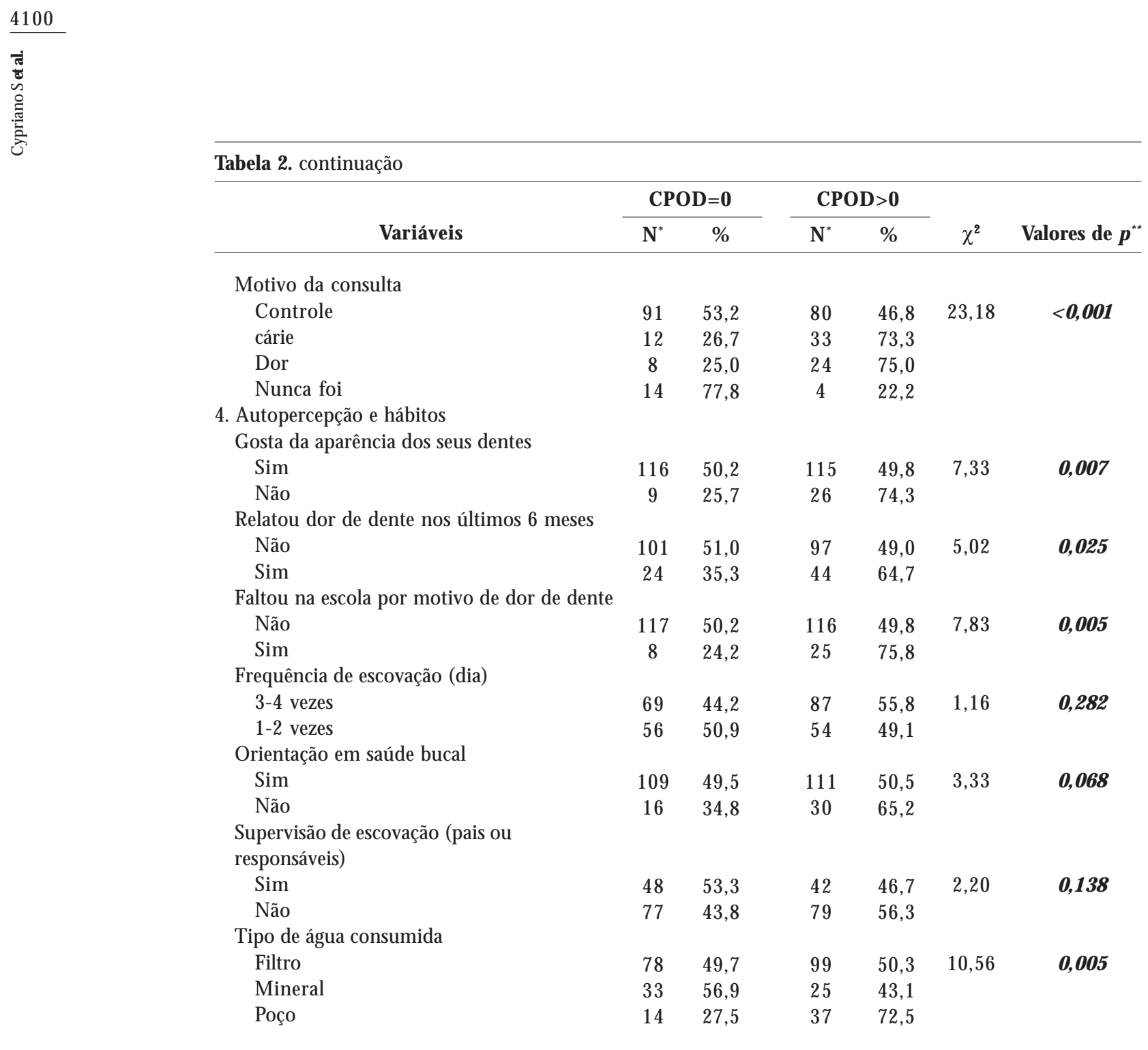

${ }^{*}$ A soma das amostras por categoria pode ser inferior a 266, devido ao não preenchimento do campo (informação perdida). **alor dep: uncorrected

Um outro aspecto metodológico quemerece destaque se refere à alta taxa de resposta, pois sabe se das dificuldades em se obter tanto as autorizações para o exame, quanto os questionários preenchidos corretamente, conforme apontado por Mello eAntunes ${ }^{33}$.

Uma das limitações desteestudo équea renda familiar e/ou do responsável pela família não foi avaliada. Durante o estudo piloto algumas pessoas se sentiram constrangidas em responder esta questão, não obtendo respostas em aproximadamente $65 \%$ dos questionários. Optou-se assim por avaliar o nível socioeconômico de forma indireta, através de outras variáveis. Entretanto como a renda familiar mostra-se como um importantefator associado à cárie dentária ${ }^{8,34}$, devese buscar uma outra forma de abordagem para se obter informações confiáveis nesse sentido.
No presente estudo, a análise de regressão logística multivariada identificou quatro variáveis estatisticamente significantes em relação ao desfecho estudado: gostar da aparência dos dentes, motivo da consulta, profissão do responsável pela família e o tipo de escola. Estes resultados se referem a uma localidade de baixa prevalência de cáriee devem ser interpretados considerando as características inerentes à população estudada, pois trata-se de uma localidade com alto nível de desenvolvimento humano. 0 IDH é considerado um potente medidor das condições sociais eambientais, refletindo a renda ea educação, além de indicadores extremamentesensíveis como a mortalidade infantil. Sendo inclusive demonstrado por alguns autores que existe uma correlação entre os altos valores do IDH ebaixos valores de experiência de cárie ${ }^{35,36}$, interferindo, 
Tabela 3. Análise univariada das variáveis que apresentaram significância estatística. Número, porcentagens, OR, intervalos de confiança e valores de $p$ em escolares de 12 anos de idade. Jundiaí (SP), 2003.

\begin{tabular}{|c|c|c|c|c|c|c|}
\hline \multirow[b]{2}{*}{ Variáveis } & \multicolumn{2}{|c|}{$C P O D=0$} & \multicolumn{2}{|c|}{$C P O D>0$} & \multirow[b]{2}{*}{ OR [IC 95\%] } & \multirow[b]{2}{*}{$P^{* *}$} \\
\hline & $\mathrm{N}^{*}$ & $\%$ & $\mathrm{~N}^{*}$ & $\%$ & & \\
\hline \multicolumn{7}{|l|}{ 2. Nível socioeconômico } \\
\hline \multicolumn{7}{|l|}{ Tipo de escola } \\
\hline Privada & 44 & 62,9 & 26 & 37,1 & 1 & \\
\hline Pública & 81 & 41,3 & 115 & 58,7 & $2,40[1,37 ; 4,22]$ & 0,0022 \\
\hline \multicolumn{7}{|l|}{ Localização da escola } \\
\hline Urbana & 111 & 49,8 & 112 & 50,2 & 1 & \\
\hline Rural & 14 & 32,9 & 29 & 67,4 & $2,05[1,03 ; 4,09]$ & 0,0410 \\
\hline \multicolumn{7}{|l|}{ Tipo de M oradia } \\
\hline Própria & 83 & 51,6 & 78 & 48,4 & 1 & \\
\hline Alugada & 20 & 51,3 & 19 & 48,7 & $1,01[0,50 ; 2,04]$ & 0,9758 \\
\hline Cedida & 22 & 33,3 & 44 & 66,7 & $2,13[1,17 ; 3,87]$ & 0,0133 \\
\hline \multicolumn{7}{|l|}{$\mathrm{N}^{\circ}$ de cômodos no domicilio } \\
\hline$>6$ & 46 & 58,2 & 33 & 41,8 & 1 & \\
\hline$<6$ & 79 & 42,2 & 108 & 57,8 & $1,91[1,12 ; 3,25]$ & 0,0177 \\
\hline \multicolumn{7}{|l|}{$\mathrm{N}^{\circ}$ de aparelhos de televisão } \\
\hline$>2$ & 79 & 57,7 & 58 & 42,3 & 1 & \\
\hline$<2$ & 46 & 35,7 & 83 & 64,3 & $2,46[1,50 ; 4,03]$ & 0,0004 \\
\hline \multicolumn{7}{|c|}{ Profissão do responsável pela família } \\
\hline Não manual & 54 & 60,7 & 35 & 39,3 & 1 & \\
\hline Manual & 71 & 40,1 & 106 & 59,9 & $2,30[1,37 ; 3,88]$ & 0,0017 \\
\hline \multicolumn{7}{|c|}{ Anos de estudo do responsável pela família } \\
\hline$>8$ & 59 & 56,7 & 45 & 43,3 & 1 & \\
\hline$<8$ & 66 & 40,7 & 96 & 59,3 & $1,91[1,16 ; 3,14]$ & 0,0112 \\
\hline \multicolumn{7}{|c|}{ 3.Acesso a serviços de Saúde Bucal } \\
\hline \multicolumn{7}{|c|}{ Tipo de tratamento } \\
\hline Privado/Convênio & 90 & 55,6 & 72 & 44,4 & 1 & \\
\hline Público & 35 & 33,7 & 69 & 66,3 & $2,46[1,48 ; 4,11]$ & 0,0005 \\
\hline \multicolumn{7}{|l|}{ M otivo da consulta } \\
\hline Controle & 91 & 53,2 & 80 & 46,8 & 1 & \\
\hline Cárie/Dor & 20 & 26,0 & 57 & 74,0 & $3,24[1,80 ; 5,86]$ & 0,0001 \\
\hline \multicolumn{7}{|c|}{ 4. Autopercepção e hábitos } \\
\hline \multicolumn{7}{|c|}{ Gosta da aparência dos seus dentes } \\
\hline Sim & 116 & 50,2 & 115 & 49,8 & 1 & \\
\hline Não & 9 & 25,7 & 26 & 74,3 & $2,91[1,31 ; 6,49]$ & 0,0088 \\
\hline \multicolumn{7}{|c|}{ Relatou dor de dente nos últimos 6 meses } \\
\hline Não & 101 & 51,0 & 97 & 49,0 & 1 & \\
\hline Sim & 24 & 35,3 & 44 & 64,7 & $1,91[1,08 ; 3,38]$ & 0,0262 \\
\hline \multicolumn{7}{|c|}{ Faltou na escola por motivo de dor de dente } \\
\hline Não & 117 & 50,2 & 116 & 49,8 & 1 & \\
\hline Sim & 8 & 24,2 & 25 & 75,8 & $3,15[1,37 ; 7,28]$ & 0,0072 \\
\hline \multicolumn{7}{|l|}{ Tipo de água consumida } \\
\hline Filtro/M ineral & 111 & 51,6 & 104 & 48,4 & 1 & \\
\hline Poço & 14 & 27,5 & 37 & 72,5 & $2,82[1,44 ; 5,52]$ & 0,002 \\
\hline
\end{tabular}

${ }^{*}$ A soma das amostras por categoria pode ser inferior à 266, devido ao não preenchimento do campo (informação perdida).

possivelmente, com os fatores determinantes da doençacárie.

As variáveis como gênero, etnia e residir no município desdeo nascimento não mostraram significância em relação ao desfecho estudado, resul- tado este suportado pelos achados de Okullo et al. ${ }^{37}$. Entretanto, sabe-se que o irrompimento dos dentes ocorre, mais frequentemente, primeiro no sexo feminino e, dependendo da idadeanalisadae/ ou nível de experiência de cárie, pode-seencontrar 
Tabela 4. Análise de regressão logística multivariada. Modelo final para os fatores associados à experiência de cárie em crianças de 12 anos de idade. Jundiaí, 2003.

\begin{tabular}{|c|c|c|c|c|}
\hline Variáveis & Coeficiente & OR & IC $(95 \%)$ & $\mathrm{p}$ \\
\hline \multicolumn{5}{|c|}{ Gosta da aparência dos seus dentes } \\
\hline Sim & referência & 1,00 & 1,$56 ; 10,16$ & 0,004 \\
\hline Não & 1,3812 & 3,98 & & \\
\hline \multicolumn{5}{|l|}{ M otivo da consulta } \\
\hline Controle & referência & 1,00 & 1,$33 ; 4,64$ & 0,004 \\
\hline Cárie/Dor & 0,9079 & 2,48 & & \\
\hline \multicolumn{5}{|c|}{ Profissão do responsável pela família } \\
\hline Não manual & referência & 1,00 & 1,$09 ; 3,53$ & 0,025 \\
\hline Manual & 0,6733 & 1,96 & & \\
\hline \multicolumn{5}{|l|}{ Tipo de escola } \\
\hline Privada & referência & 1,00 & 1,$18 ; 4,18$ & 0,014 \\
\hline Pública & 0,7966 & 2,22 & & \\
\hline
\end{tabular}

$-2 \log$ likelihood $=301.857(p<0,0001)$

diferenças quanto ao gênero em função do risco relacionado ao tempo de exposição dos dentes.

Sendo assim, quanto aos fatores de nível socioeconômico investigados, duas variáveis foram associadas significativamente com a ocorrência da cárie dentária: estudar em escola pública e baixa qualificação profissional do responsável pela família. Os estudantes de escolas privadas apresentaram menor prevalência eseveridade de cárie, estando coerentes com o padrão observado em escolares do município de João Pessoa e do interior do Estado de São Paulo ${ }^{4,38}$, ede crianças em idade préescolar de Blumenau ${ }^{39}$, com a ressalva de que nestes dois últimos estudos ape nas procedeu-se a análise descritiva da cárie. I rigoyen et al. ${ }^{9}$ ao compararem a experiência de cárie na dentição permanente de crianças de escolas públicas e privadas da cidade do M éxico verificaram que as crianças de escolas públicas tem 1,24 vezes mais chance de pertencer ao grupo de alto risco $(C P O D>3)$, sendo também maior a experiência e as necessidades de tratamento.

Por outro lado, alguns estudos não encontraram diferenças entre os grupos ${ }^{18,19,40,41}$, mesmo se tratando de amostras maiores do que a do presente estudo. Neste, as crianças do ensino público apresentaram uma chance 2,2 vezes maior de ter experiência de cárie, quando comparadas àquelas que estudam em escolas privadas, sendo este resultado suportado pela análise de regressão logística multivariada, possibilitando uma melhor compreensão dos fatores associados. Os resultados também sugerem que as condições econômicas e sociais podem ser limitadoras em relação à adoção de hábitos saudáveis, refletindo em uma maior prevalência de doença, mesmo em um município com alto IDH. Ressalta-se aqui que a amostra foi composta por escolares tanto do ensino público quanto do privado de undiaí, sendo representativa para este município eque inferências diretas para outros municípios com IDH similar podem ser realizadas com esta ressalva, levar em consideração a prevalência de cárie dentária e, principalmente, os níveis socioeconômicos destemunicípio.

A profissão do responsável pela família também se relacionou significativamente com a experiência de cárie dentária, ou seja, os níveis de saúde bucal em relação à cáriesão melhores quando o responsável pela família possui uma meIhor qualificação profissional, tendo concordância com os estudos de Cortellazzi et al. ${ }^{42}$, Källestål e Wall ${ }^{5}$ e Nieto Garcia et al. ${ }^{43}$, mostrando uma forma bastante comum e objetiva para medir o nível socioeconômico e um bom indicador para a nossa realidade, pois diferenciou os padrões de saúde-doença.

Peres et al. ${ }^{8}$ investigaram a escolaridade do pai e da mãe da criança, e a do primeiro quando alta, mostra-se associada com baixa prevalência de cárie $(p<0,05)$, mas esta variável também não permaneceu no modelo final, após a análise de regressão, em concordância com os resultados da presente pesquisa em quese investigou os anos de estudo do responsável pela família, que também se mostrou associado com a experiência de cárie. Segundo afirmam Shou e Uitenbroek ${ }^{44} \mathrm{e}$ Verrips et al. ${ }^{45}$, o grau de instrução elevado possibilita maiores oportunidades de acesso à informação sobre saúde e as crianças que convivem com adultos nessa condição estão sujeitas a hábitos e condutas de saúde bucal mais saudáveis. 
Em acréscimo, Van Nieuwenhuysen et al..$^{6} \mathrm{em}$ um estudo de coorte realizado em crianças da Bélgica aos 12 anos de idade, não apenas constatou queas crianças, de grupo socialmentemais privilegiado em relação à educação eà profissão dos pais, apresentaram níveis menores de CPOD, como também foi maior a redução de cárie durante 0 período analisado, quando se comparou com o grupo de crianças menos privilegiadas. Os determinantes sociais podem exercer um diferencial tão forte que mesmo quando as crianças têm acesso a um sistema público e universal de saúde bucal, estas diferenças não são eliminadas, conformeverificaram Ismail e Woolsung Sohn ${ }^{46}$, o que possivelmentedeve ocorrer éuma redução dasiniquidades no acesso a cuidados em saúde bucal.

A importância dos fatores sociais na evolução da cárie dentária tem sido cada vez melhor elucidada em relatos científicos, demonstrando que as condições sociais têm uma determinação importante nas desigual dades da distribuição da cárie dentária $a^{7,10,11,14,47-49}$, sendo também confirmados pelos achados do presente estudo.

Apesar da estatística descritiva da cárie ter demonstrado piores níveis de saúde bucal nas escolas rurais, em comparação com as urbanas, em concordância com os achados de O kullo et al. ${ }^{37}$, queverificaram estepadrão em duas comunidades de Uganda (África), em escolares entre 13 e 19 anos de idade, esta variável não se mostrou um fator associado à cárie dentária no modelo final do presente estudo.

Há al guns relatos na literatura de que maiores escores do CPOD são frequentemente registrados em áreas urbanas de países em desenvolvimento ${ }^{5,50}$. Muito possivelmente, fatores relacionados ao nível socioeconômico das famílias investigadas devem exercer um efeito confundidor sobre esta variável, sendo constatado por El$N$ adeef et al. ${ }^{51}$, que ao comparar a distribuição da cárie dentária em crianças de diferentes estratos na Nigéria, observou que as crianças da área urbana e semiurbana apresentaram um maior risco de cárie do que as crianças da zona rural, mas que ao ajustar pela classe social na análise, a urbanização não apresentou mais este efeito.

Não se verificou uma associação entre o número de pessoas residentes na casa e a experiência de cárie, corroborando com os achados de Peres et al. ${ }^{8}$, com a ressalva de que neste estudo de Florianópolis, investigou-se a alta severidade da cárie. M uito provavelmente a forma de coleta desta informação necessite ser revista, pois tem sido utilizada a aglomeração domiciliar (porcentagem da população vivendo em domić́lios com mais de dois moradores por dormitório), encontrando correlações positivas entre níveis piores de saúde e maior aglomeração ${ }^{13}$.

Quanto às variáveis relacionadas ao acesso a serviços odontológicos, no presente estudo, apenas o motivo da consulta foi significantemente associado com a experiência de cárie, em concordância com os achados de Van Nieuwenhuysen et al. ${ }^{6}$. Cangussú et al. ${ }^{40}$ ao estudar a experiência de cárie em escolares de escolas públicas e privadas da Bahia, de 12 e 15 anos de idade, não encontrou diferenças na média do CPOD eno percentual de crianças livres de cárie entre os dois grupos e apenas a variável acesso ao dentista no último ano mostrou-se associada às diferenças encontradas. Afirma que grupos com melhores níveis socioeconômicos têm apresentado maior regularidade no acesso a bens de consumo e a serviços de saúde. Assim, acredita-se que em futuros estudos esta variável deva ser melhor investigada pois no presente não se tratou da frequência do acesso à tratamento em saúde bucal e sim se a criança o teve.

Outro aspecto que merece ser discutido se refereao percentual decrianças quenunca foram ao dentista. No presente estudo, este foi de cerca de $12 \%$, e segundo Barros e Bertoldi ${ }^{2} 2$ há um diferencial bastantepronunciado nautilização de serviços em relação ao grupo de maior e menor poder aquisitivo, pois no grupo de maior renda apenas $5 \%$ das crianças aos 12 anos nunca foram ao dentista. Essa proporção aumenta para $40 \%$ entre os menos favorecidos economicamente. Entretanto esta variável precisa ser interpretada com cautela, pois maior possibilidade deacesso aos serviços pode não estar relacionada com a redução de cárie dentária, conforme demonstrado por Baldani et al. ${ }^{11}$.

Finalmente as questões que foram avaliadas com relação à autopercepção também apresentaram associações plausíveis com o desfecho estudado. A experiência de cárie dentária foi associada ao fato da criança não gostar da aparência dos seus dentes $(O R=3,98)$, sugerindo que 0 autocuidado e/ou práticas mais saudáveis de saúde bucal podem não estar incorporadas no dia a dia destas crianças, ou se estiverem, não são val orizadas. Este achado tanto podeser útil na identificação de crianças com maior risco de desenvolver cárie, somando-se ao elenco das variáveis para triagem de risco, como também aponta que a autoestima é um aspecto fundamental a ser trabalhado nas estratégias de promoção de saúde.

A hipótese da causalidade reversa, em estudos transversais, não permite estabelecer se a ex- 
posição precedeu ou não o desfecho. Somente estudos de acompanhamento permitem determinar com melhor exatidão a temporalidade entre causa e efeito e, desse modo, os resultados do presente estudo devem ser interpretados com os devidos cuidados. Assim, a relação entreo fato da criança não gostar da aparência dos seus dentes e a experiência de cárie representam um viés do estudo, como os demais fatores associados ao $C P O D>0$.

A frequência deescovação diária não foi associada com a experiência de cárie. A categorização adotada deve-se ao fato de que não houve respostas em que este número tenha sido inferior a uma vez ao dia. Ismail e Woosung Shon ${ }^{46}$ encontraram esta associação, sendo detectado melhores índices de saúde bucal quando as crianças escovavam os seus dentes 1 ou 2 vezes ao dia, em comparação com as que escovavam menos do que uma vez ao dia. Questiona-se um possível viés nas respostas dos pais no presente estudo, pois mais de $63 \%$ dos mesmos não supervisionam a escovação dos seus filhos, visto que são indiscutíveis os benefícios desta prática, pois, conforme demonstraram Adair et al..$^{53}$, a habilidade dos pais no controle da escovação e do consumo de açúcar são os preditores mais significantes de hábitos favoráveis em crianças, refletindo em melhores condições de saúde bucal.

Os resultados do presente estudo permitem apontar que o nível socioeconômico, o acesso aos serviços e a autopercepção se relacionaram com a experiência de cárie dentária em crianças aos 12 anos de idade, sendo fundamental que medidas efetivas de promoção de saúde bucal sejam implementadas para possibilitar que as diferenças nos níveis de saúde observadas sejam reduzidas. As medidas de promoção de saúde, além de estimular boas práticas, deveriam estabelecer estratégias para favorecer 0 autocuidado e a auto-estima das crianças.

Recomenda-se ainda que, em futuros estudos, a renda familiar seja também avaliada, pois mostra-se como um importante fator associado à cárie dentáriå, além de outros fatores biopsicossociais relacionados a multifatoriedade da mesma.

Assim, estudar os fatores associados à cárie dentária em localidades onde esta apresente baixa incidência, em países em desenvolvimento, como é o caso do Brasil, torna-se uma questão de importância crescente.

\section{Colaboradores}

Todos os autores participaram igualmente de todas as etapas da elaboração do artigo.

\section{Agradecimentos}

Os autores agradecem à colaboração das crianças e dos responsáveis por autorizarem a realização dos exames e terem respondido ao questionário. Em especial a toda a equipe de examinadores e anotadores que realizaram os exames epidemiológicos, possibilitando queesta pesquisa fosse realizada. Agradecemos também a Juliana Balbinot H ilgert, pelo auxílio na análise estatística dos resultados e à M aria Paula Rando M eirelles pela digitação dos dados. 
Referências

1. Pattussi MP, Marcenes W, Croucher R, Sheiham A. Social deprivation, income cohesion and dental caries in Brazilian school children. Soc Sci M ed 2001; 53(7):915-925.

2. Almeida-Filho N, Kawachi I, Pellegrini Filho A, Dachs NW. Research on health inequalities in Latin America and the Caribbean: bibliometric analysis (1971-2000) and descriptive content analysis (19711995). Am J Public Health 2003; 93(12):2037-2043.

3. Rihs LB, Gushi LL, Sousa MLR, Wada RS. Cárie Dentária Segundo o nível socioeconômico em Itapetininga - SP. Rev Odonto Ciênc 2005; 20(50):367371.

4. Hoffmann RHS, Cypriano S, Sousa MLR, Wada RS. Experiência de cárie dentária em crianças de escolas públicas e privadas de um município com água fluoretada. Cad Saude Publica 2004; 20(2):522-528.

5. Källestål C, Wall S. Socio-economic effect on caries. Incidence data among Swedish 12-14-year-old. Community Dent Oral Epidemiol 2002; 30(2):108-114.

6. Van Nieuwenhuysen JP, Carvalho JC, D'Hoore W. Caries reduction in Belgian 12-year-old children related to socioeconomic status. Acta O dontol Scand 2002; 60(2):123-128.

7. Gillcrist JA, Brumley DE, Blackford JU. Community socioeconomic status and children's dental health. J Am Dent Assoc 2001; 132(2):216-222.

8. Peres KGA, Bastos JRM, Latorre M RDO. Severidade de cárie em crianças e relação com aspectos sociais e comportamentais. Rev Saude Publica 2000; 34(4):402-408.

9. Irygoyen ME, Maupomé G, M ejía AM. Caries experience and treatment needs in a 6- to 12-year-old urban population in relation to socio-economic status. Community Dent Health 1999; 16(4):245-249.

10. Antunes JLF, Narvai PC, Nugent ZJ. Measuring inequalitities in the distribution of dental caries. Community Dent Oral Epidemiol 2004; 32(1):41-48.

11. Baldani MH, Vasconcelos AGG, Antunes JLF. Associação do índice CPO-D com indicadores sócioeconômicos e de provisão de serviços odontológicos no Estado do Paraná, Brasil. Cad Saude Publica 2004; 20(1):143-152.

12. Antunes JLF, Frazão P, Bispo CM, Pegoretti T. Spatial analysis to identify differentials in dental needs by area-based measures. Community Dent Oral Epidemiol 2002; 30(2):133-142.

13. Baldani MH, Narvai PC, Antunes JLF. Cárie Dentária e condições sócio-econômicas no Estado do Paraná, Brasil, 1996. Cad Saude Publica 2002; 18(3): 155-163.

14. Pattussi M P, Marcenes W, Croucher R, Sheiham A Social deprivation, income inequality, social cohesion and dental caries in Brasilian school children. Soc Sci M ed 2001; 53(7):915-925.

15. Truin GJ, König KG, Bronkhorst EM, Frankenmolen $F$, Mulder J, van't Hof MA. The trends in caries experience of 6- and 12-year-old children of different socioeconomic status in the Hague. Caries Res 1998; 32(1):1-4

16. Laloo R, Myburgh NG, Hodbell MH. Oral Health, socio-economic development and national oral health policies. Int Dent J 1999; 49(4):196-202.
17. Ellwood RP, Mullane DM. Identification of areas with hight leves of untreated dental caries. Community Dent Oral Epidemiol 1996; 24(1):1-6.

18. Taani DQ. Relationship of socioeconomic background to oral hygiene, gingival status, and dental caries in children. Quintessence Int 2002; 33(3):195198.

19. Witt MCR. Pattern of caries experience in a 12-year old Brasilian population related to socioeconomic background. Acta O dontol Scand 1992; 50(1):25-30.

20. Bastos JLD, Nomura LH, Peres MA. Tendência de cárie dentária em escolares de 12 e 13 anos de idade de uma mesma escola no período de 1971 a 2002, em Florianópolis, Santa Catarina, Brasil. Cad Saude Publica 2004; 20(1):117-122.

21. Carvalho JC, Van Nieuwenhuysen JP, D'Hoore W. The decline in dental caries among Belgian children between 1983 and 1998. Community Dent Oral Epidemiol 2001; 29(1):55-61.

22. Dini EL, Foschini ALR, Brandão IMG, Silva SRC. Changes in caries prevalence in 7-12 year-old children from Araraquara, São Paulo, Brazil: 1989-1995. Cad Saude Publica 1999; 15(3):617-621.

23. Cypriano S, Duran AI, Sousa M LR, Wada RS. Dental caries experience in 12-year-old schoolchildren in southeastern Brazil. J Appl Oral Sci 2008; 16(4):286-292.

24. Martins RJ, Garbin CAS, Garbin, AJI, M oimaz SAS, Saliba O. Declínio da cárie em um município da região noroeste do Estado de São Paulo, Brasil, no período de 1998 a 2004. Cad Saude Publica 2006; 22(5):1035-1041.

25. Gomes PR, Costa SC, Cypriano S, Sousa M LR. Paulínia, São Paulo, Brasil: situação da cárie dentária com relação às metas OM S 2000 e 2010. Cad Saude Publica 2004; 20(3):866-870.

26. Birkeland JM, Haugejorden 0 , von der Fehr FR. Analyses of the caries decline and incidence among Norwegian adolescents 1985-2000. Acta Odontol Scand 2002; 60(5):281-289.

27. Fundação Sistema Estadual de Análise de Dados, 2004 (SEADE). [acessado 2004 maio 23]. Disponível em: http://www.seade.gov.br/perfil/index.html

28. Cypriano S, Duran AI, Sousa M LR, Wada RS. Metas da Organização M undial da Saúde para o ano de 2000 e a saúde bucal na região de Campinas, Brasil. Arq O dontol 2002; 38(2):151-162.

29. Silva N N. Amostragem Probabilística: um curso introdutório. São Paulo: Editora da Universidade de São Paulo; 1998.

30. Brasil. M inistério da Saúde (M S). Projeto SB 2000. Condições de saúde bucal da população brasileira no ano 2000. Manual do Examinador. Brasília: Ministério da Saúde; 2001.

31. Petersen PE. Society and oral health. In: Pine CM. Community Oral Health. Oxford: Wright; 1997. p. 20-54.

32. World Health Organization (WHO). Oral Health Surveys, basic methods. 4th ed. Geneve: WH O; 1997.

33. M ello TRC, Antunes JLF. Prevalência de cárie dentária em escolares da região rural de Itapetininga, São Paulo, Brasil. Cad Saude Publica 2004; 20(3):829835. 
34. Peres MA, Latorre MRDO, Sheiham A, Peres KG, Barros FC, Hernandez PG. Determinantes sociais e biológicos da cárie dentária em crianças de 6 anos de idade: um estudo transversal aninhado numa coorte de nascidos vivos no sul do Brasil. Rev Bras Epidemiol 2003; 6(4):293-306.

35. Frias AC, Antunes JLF, Junqueira SR, Narvai PC. Determinantes individuais e contextuais da prevalência de cárie dentária não tratada no Brasil. Rev Panam Sauld Publica 2007; 22(4):279-285.

36. Moysés, S.J. Desigualdades em saúde bucal e desenvolvimento humano: um ensaio em preto, branco e alguns tons de cinza. Rev Bras Odontol Saúde Coletiva 2000; 1(1):7-17.

37. Okullo I, Nordrehaug A, Haugejorden O, Mugisha Rwenyonyi C. Variation in caries experience and sugar intake among secondary school students in urban and rural U ganda. Acta O dontol Scand 2003; 61(4):197-202.

38. M oreira PVL, Rosenblatt $A$, Passos IA. Prevalência de cárie em adolescentes de escolas públicas e privadas na cidade de João Passoa, Paraíba, Brasil. Cien Saude Colet 2007; 12(5):1229-1236.

39. Traebert JL, Peres MA, Galesso ER, Zabot NE, $M$ arcenes W. Prevalência e severidade da cárie dentária em escolares de seis a doze anos de idade. Rev Saude Publica 2001; 35(3):283-288.

40. Cangussu MCT, Castellanos RA, Pinheiro M F, Albuquerque SR, Pinho C. Cárie dentária em escolares de 12 e 15 anos de escolas públicas e privadas de Salvador, Bahia, Brasil, em 2001. Pesqui O dontol Bras 2002; 16(4):379-384.

41. Narvai PC, Castellanos RA, Frazão P. Prevalência de cárie em dentes permanentes de escolares no município de São Paulo, SP, 1970-1996. Rev Saude Publica 2000; 34(2):196-200.

42. Cortelazzi KL, Tagliaferro EPS, Assaf AV, Tafner APM F, Ambrosano GM B, Bittar TO, Meneghim MC, Pereira AC. Influência de variáveis socioeconômicas, clínicas e demográficana experiência de cárie dentária em pré-escolares de Piracicaba, SP. Rev Bras Epidemiol 2009; 12(3):490-500.

43. Nieto Garcia VM, Nieto Garcia MA, Lacalle Remigio JR, Abdel-Kader M artin L. Oral health of school children in Ceuta. Influences of age, sex, ethnic, background and socioeconomic level. Rev Esp SaIud Publica 2001; 75(6):541-549.

44. Shou L, Uitenbroek D. Social and behavioural indicators of caries experience in 5-year-old children. Community Dent Oral Epidemiol 1995; 23(5):276-81.
45. Verrips GH, Kaisbeek H, Eikman MAJ. Ethnicity and maternal education as risk indicators for dental caries, and the role of dental behavior. Community Dent Oral Epidemiol 1993; 21(4):209-214.

46. Ismail Al, Woosung Shon. The impact of universal access to dental care on disparities in caries experience in children. J Am Dent Assoc 2001; 132(3):295303.

47. M arthaler TM. Changes in Dental Caries 1953-2003. Caries Res 2004; 38(3):173-181.

48. Peres MA, Peres KG, Antunes JLF, Junqueira SR, Frazão $P, N$ arvai $P C$. The association between socioeconomic development at the town level and the distribution os dental caries in Brasilian children. Rev Panam Salud Publica 2003; 14(3):149-157.

49. Locker D. Deprivation and oral health: a review. Community Dent Oral Epidemiol 2000; 28(3):161-169.

50. Petersen PE, Aarhus NH, Pooviset N, Prommajan J, Watanapa A. Oral health status and oral health behavior of urban and rural schoolchildren in Southern Thailand. Int Dent J 2001; 51(2):95-102.

51. El-Nadeef MAI, Adegbembo AO, Honkala E. The association of urbanisation with the prevalence of dental caries among schoolchildren in Nigeria new capital territory. Int Dental J 1998; 48(1):44-49.

52. Barros AJD, Bertoldi AD. Desigualdades na utilização e no acesso a serviços odontológicos: uma avaliação em nível nacional. Cien Saude Colet 2002; 7(4):709-717.

53. Adair MP, Pine CM, Girvan B, Nicoll AD, Gillet $A$, Anwar S. Broukal Z, Chestnutt IG, Declerk D, Feng $X P$, Ferro R, Freeman R, Grant-M ills D, Gugushe $T$, Hunsrisakhun J, Irigoyen-Camacho M, Lo ECM, Mohamed HM, Naidoo S, Nyandindi U, Vibeke JP, Ramos-Gomez F, Razanamihaja N, Shahid S, M arit SS, Skur OP, Splieth C, Teo CS, Whelton H, Young DW. Familial and cultural perceptions and beliefs of oral hygiene and dietary practices among ethnically and socio-economically diverse groups. Community Dental Health 2004; 21(Supl.):102-111.

Artigo apresentado em 30/08/2010

Aprovado em 12/12/2010

Versão final apresentada em 05/01/2011 